\section{Assessment of myocardial ischaemia using tissue Doppler imaging in pseudoexfoliation syndrome}

N Demir', T Ulus², OE Yucel', ET Kumral'1, E Singar ${ }^{1}$ and $\mathrm{HI}$ Tanboga $^{2}$

\section{Abstract}

Purpose Pseudoexfoliation (PEX) syndrome is characterized by the widespread deposition of abnormal extracellular fibrillary material on many ocular and extraocular tissues. We aimed to investigate the association between PEX syndrome and subclinical myocardial ischaemia, using tissue Doppler echocardiography. Methods Thirty-two patients with pseudoexfoliation syndrome (mean age: $66 \pm 9$ years, 22 men) and 25 healthy individuals (mean age: $67 \pm 8$ years, 13 men) were included in the study. Patients with overt coronary artery disease, congestive heart failure, valvular heart disease, cardiomyopathy, and left ventricular hypertrophy were excluded from the study. Tissue Doppler imaging was performed at the septal, lateral, anterior, and inferior mitral annuluses. Differences between the groups were evaluated by the unpaired $t$-test and the Mann-Whitney $U$-test, with a $P$-value of $<\mathbf{0 . 0 5}$ considered significant. Results Baseline clinical characteristics, twodimensional, and Doppler echocardiography parameters were similar in the PEX and control groups. Peak systolic velocities at the septal, lateral, anterior, and inferior annuluses were significantly lower in patients with PEX syndrome $(P<0.001,0.01,0.02$, and 0.02 , respectively). The early diastolic velocity at the septal annulus, and the ratio of early/late diastolic velocity at the lateral annulus were significantly lower in the study group $(P=0.03)$.

Conclusion PEX syndrome is a common disorder of extracellular matrix. Our data suggest that there may also be an association between PEX syndrome and subclinical myocardial ischaemia in patients who have no signs and symptoms of ischaemia. Thus, we think that ophthalmologists should consider informing their PEX syndrome patients' general practitioners about a possible cardiac risk.

Eye (2011) 25, 1177-1180; doi:10.1038/eye.2011.145; published online 24 June 2011

Keywords: exfoliation syndrome; myocardial perfusion imaging; myocardial ischaemia

\section{Introduction}

Pseudoexfoliation (PEX) syndrome is an age-related disorder and the most common abnormal finding associated with chronic open-angle glaucoma. ${ }^{1,2}$ It is characterized by progressive accumulation of abnormal extracellular fibrillary material on many ocular and extraocular tissues, including the periphery of blood vessels., ${ }^{3,4}$ PEX syndrome has been associated with many cardiovascular and cerebrovascular diseases. ${ }^{4}$ In addition, it has been found that the prevalence of PEX syndrome was significantly higher in patients with documented coronary artery disease (CAD), a history of myocardial infarction (MI), or ischaemia. ${ }^{5,6}$

Tissue Doppler imaging (TDI) is a useful method for quantification of regional myocardial wall motion abnormalities related to myocardial ischaemia. ${ }^{7-9}$

In this study, we aimed to investigate whether or not subclinical myocardial ischaemia exists in patients with PEX syndrome using TDI. If this technique establishes an association between
${ }^{1}$ Department of Ophthalmology, Erzurum Regional Education and Research Hospital, The Ministry of Health, Erzurum, Turkey

${ }^{2}$ Department of Cardiology, Erzurum Regional Education and Research Hospital, The Ministry of Health, Erzurum, Turkey

Correspondence: N Demir, Department of Ophthalmology, Erzurum Regional Education and Research Hospital, The Ministry of Health, Erzurum 25070, Turkey Tel: + 905067747862 ; Fax: + 904422325090 . E-mail:drnihaldemir@ yahoo.com

Received: 6 September 2010

Accepted in revised form: 24 April 2011 Published online: 24 June 2011 
PEX syndrome and myocardial ischaemia in asymptomatic patients, it may reveal the need of careful evaluation of subclinical myocardial ischaemia in PEX patients.

\section{Materials and methods}

The study group was composed of 32 patients with PEX syndrome evaluated at Erzurum Regional Education and Research Hospital, The Ministry of Health, Erzurum, Turkey. We randomly selected the control group from the age- and sex-matched healthy subjects without PEX syndrome. Clinical, demographic characteristics, and medications were recorded. Patients with overt CAD, congestive heart failure ( $\mathrm{CHF}$; ejection fraction $<45 \%$ ), valvular heart disease or valvular surgery, cardiomyopathy, and left ventricular hypertrophy were excluded from the study (two PEX patients had CAD, one had hypertrophic $\mathrm{CMP}$, one had severe aortic valve disease, and one had moderate mitral valve disease and $\mathrm{CHF}$ ). CAD was considered in the presence of one of the following: typical angina, ST-segment or T-wave changes specific for myocardial ischaemia, pathological Q-waves on ECG, regional wall motion abnormality on echocardiography, a non-invasive stress test revealing ischaemia, or a history of $\mathrm{MI}$ or revascularization.

All the patients had complete ophthalmological examination including visual activity testing, slit-lamp examination with dilated pupils, applanation tonometry, and dilated examination of the posterior segment. The patients who had the typical greyish deposits on the anterior lens capsule and the pupillary border were included in the PEX group. ${ }^{5}$

Echocardiography was performed using a Vivid 7 ultrasound system (GE Healthcare, Chalfont St Giles, UK). Two-dimensional and transmitral velocity (E) measurements were performed according to the recommendations of the American Society of Echocardiography. ${ }^{10}$ Using pulse-wave tissue Doppler, we acquired the TDI velocities at the septal, lateral, inferior, and anterior mitral annuluses. Peak systolic velocity (S), early and late diastolic annular velocities (Ea and $\mathrm{Aa}$ ), the ratio of early and late diastolic annular velocities ( $\mathrm{Ea} / \mathrm{Aa})$, and the ratio of transmitral velocity and early diastolic annular velocity $(\mathrm{E} / \mathrm{Ea})$ were obtained.

We certify that all applicable institutional and governmental regulations concerning the ethical use of human volunteers were followed during this research.

\section{Statistical analysis}

All computations were performed by using the Statistical Program for the Social Services version 10.0. for
Windows (SPSS Inc., Chicago, IL, USA). Categorical variables were compared with $\chi^{2}$-test. The distribution of continuous variables for normality was tested with one-sample Kolmogorov-Smirnov test, and data were presented as mean \pm SD or median and interquartile ranges, as appropriate. Differences between patients with and without PEX syndrome in normally and non-normally distributed variables were evaluated by the unpaired $t$-test and the Mann-Whitney $U$-test, respectively. All $P$-values were two-sided, and a $P$-value of $<0.05$ was considered significant.

\section{Results}

Baseline clinical characteristics of the two groups were similar. Body mass index (BMI) was lower in the study group, but it was not significant $(P=0.06)$. Clinical characteristics of the patients are presented in Table 1. The EF, all the other two-dimensional and Doppler echocardiography parameters were similar. Twodimensional and Doppler echocardiography findings of the patients are summarized in Table 2 .

In the study group composed of PEX patients, the S-wave velocities were normal in 26 patients at the septal annulus, 31 patients at the lateral and inferior annuluses, and 30 patients at the anterior annulus. In the control group, the S-wave velocities were normal in 25 patients at the septal, lateral, and inferior annuluses, and 24 patients at the anterior annulus.

The S-wave velocities were significantly lower in patients with PEX syndrome than control subjects at the septal, lateral, anterior, and inferior annuluses $(P<0.001$, $0.01,0.02$, and 0.02 , respectively). In addition, the Ea velocity at the septal annulus and the ratio of Ea/Aa at the lateral annulus were significantly lower in patients with PEX syndrome ( $P=0.03$ for each one). The other tissue Doppler parameters were similar (Table 3 ).

\section{Discussion}

PEX syndrome is a disorder of the extracellular matrix, and is frequently related to severe chronic secondary

Table 1 Baseline clinical characteristics

\begin{tabular}{lccc}
\hline & PEX $(\mathrm{n}=32)$ & Controls $(\mathrm{n}=25)$ & P-values \\
\hline Age (years) & $66 \pm 9$ & $67 \pm 8$ & 0.85 \\
Gender (male) $n(\%)$ & $22(69 \%)$ & $13(52 \%)$ & 0.27 \\
BMI $\left(\mathrm{kg} / \mathrm{m}^{2}\right)$ & $26.9 \pm 4.2$ & $29.5 \pm 5.8$ & 0.06 \\
Heart rate (beats $/ \mathrm{min})$ & $76 \pm 11$ & $75 \pm 9$ & 0.68 \\
Hypertension $n(\%)$ & $12(38 \%)$ & $13(52 \%)$ & 0.29 \\
Diabetes mellitus $n(\%)$ & $1(3 \%)$ & $4(16 \%)$ & 0.15 \\
Tobacco smoking $n(\%)$ & $5(16 \%)$ & $6(24 \%)$ & 0.50 \\
\hline
\end{tabular}

Abbreviations: BMI, body mass index; PEX, pseudoexfoliation syndrome. 
Table 2 Two-dimensional and Doppler echocardiography parameters

\begin{tabular}{lccc}
\hline & $\begin{array}{c}\text { PEX } \\
(\mathrm{n}=32)\end{array}$ & $\begin{array}{c}\text { Controls } \\
(\mathrm{n}=25)\end{array}$ & P-values \\
\hline End-diastolic volume (ml) & $86 \pm 18$ & $83 \pm 21$ & 0.51 \\
End-systolic volume (ml) & $36 \pm 10$ & $33 \pm 11$ & 0.44 \\
Stroke volume (ml) & $51 \pm 9$ & $50 \pm 12$ & 0.66 \\
Ejection fraction (\%) & $59 \pm 5$ & $60 \pm 5$ & 0.47 \\
Interventricular septum (mm) & $10.6 \pm 1.6$ & $11.1 \pm 2.0$ & 0.27 \\
Posterior wall (mm) & $10.6 \pm 1.5$ & $11.0 \pm 1.6$ & 0.30 \\
Mitral E (cm/s) & $63 \pm 15$ & $63 \pm 15$ & 0.98 \\
Mitral A (cm/s) & $81 \pm 16$ & $83 \pm 18$ & 0.62 \\
E/A & $0.8 \pm 0.2$ & $0.7 \pm 0.1$ & 0.61 \\
\hline
\end{tabular}

Abbreviation: PEX, pseudoexfoliation syndrome.

Table 3 Tissue Doppler parameters

\begin{tabular}{|c|c|c|c|}
\hline & $\begin{array}{c}P E X \\
(\mathrm{n}=32)\end{array}$ & $\begin{array}{l}\text { Controls } \\
(\mathrm{n}=25)\end{array}$ & P-values \\
\hline Septal S (cm/s) & $6.8 \pm 1.3$ & $8.1 \pm 1.2$ & $<0.001$ \\
\hline Septal Ea $(\mathrm{cm} / \mathrm{s})$ & $7.3 \pm 1.9$ & $8.5 \pm 2.4$ & 0.03 \\
\hline Septal Aa (cm/s) & $11.3 \pm 2.6$ & $10.9 \pm 2.1$ & 0.50 \\
\hline Septal IVC (cm/s) & $5.6 \pm 2.3$ & $6.6 \pm 1.9$ & 0.08 \\
\hline Septal E/Ea (cm/s) & $9.1 \pm 3.2$ & $7.9 \pm 3.0$ & 0.14 \\
\hline Septal Ea/Aa (cm/s) & $0.7 \pm 0.2$ & $0.8 \pm 0.2$ & 0.10 \\
\hline Lateral S (cm/s) & $7.8 \pm 1.6$ & $8.9 \pm 1.5$ & 0.01 \\
\hline Lateral Ea $(\mathrm{cm} / \mathrm{s})$ & $8.7 \pm 2.4$ & $9.9 \pm 2.5$ & 0.07 \\
\hline Lateral Aa (cm/s) & $12.1 \pm 2.8$ & $11.3 \pm 2.6$ & 0.25 \\
\hline Lateral IVC (cm/s) & $6.2 \pm 2.0$ & $6.7 \pm 2.0$ & 0.33 \\
\hline Lateral E/Ea $(\mathrm{cm} / \mathrm{s})$ & $7.7 \pm 2.0$ & $6.8 \pm 2.4$ & 0.15 \\
\hline Lateral Ea/Aa $(\mathrm{cm} / \mathrm{s})$ & $0.7 \pm 0.2$ & $0.9 \pm 0.3$ & 0.03 \\
\hline Anterior S (cm/s) & $7.5 \pm 1.8$ & $8.6 \pm 1.4$ & 0.02 \\
\hline Anterior Ea $(\mathrm{cm} / \mathrm{s})$ & $8.0 \pm 2.5$ & $8.2 \pm 1.7$ & 0.79 \\
\hline Anterior $\mathrm{Aa}(\mathrm{cm} / \mathrm{s})$ & $11.3 \pm 2.3$ & $10.5 \pm 2.3$ & 0.17 \\
\hline Anterior IVC (cm/s) & $6.6 \pm 1.6$ & $7.0 \pm 1.7$ & 0.41 \\
\hline Anterior E/Ea $(\mathrm{cm} / \mathrm{s})$ & $8.6 \pm 3.0$ & $7.9 \pm 2.0$ & 0.32 \\
\hline Anterior $\mathrm{Ea} / \mathrm{Aa}(\mathrm{cm} / \mathrm{s})$ & $0.7 \pm 0.3$ & $0.8 \pm 0.2$ & 0.42 \\
\hline Inferior S (cm/s) & $7.9 \pm 1.4$ & $8.8 \pm 1.2$ & 0.02 \\
\hline Inferior Ea $(\mathrm{cm} / \mathrm{s})$ & $7.8 \pm 2.5$ & $8.7 \pm 2.3$ & 0.20 \\
\hline Inferior Aa $(\mathrm{cm} / \mathrm{s})$ & $12.9 \pm 2.9$ & $12.4 \pm 2.5$ & 0.50 \\
\hline Inferior IVC (cm/s) & $7.0 \pm 2.4$ & $7.6 \pm 1.9$ & 0.29 \\
\hline Inferior E/Ea $(\mathrm{cm} / \mathrm{s})$ & $8.8 \pm 3.4$ & $7.6 \pm 2.4$ & 0.18 \\
\hline Inferior Ea/Aa (cm/s) & $0.6 \pm 0.2$ & $0.7 \pm 0.2$ & 0.21 \\
\hline
\end{tabular}

Abbreviations: Aa, peak late diastolic velocity; Ea, peak early diastolic velocity; IVC, isovolumic contraction velocity; PEX, pseudoexfoliation syndrome; S, peak systolic velocity.

open-angle glaucoma and cataract. ${ }^{4}$ The prevalence of PEX syndrome increases markedly with age, and may affect up to $30 \%$ of people older than 60 years worldwide. ${ }^{4}$ This syndrome is characterized by deposits of white, fibrillary material on the anterior chamber structures, including the trabecular meshwork.

PEX material has also been found in connective tissue portions of visceral organs, often in the periphery of blood vessels. ${ }^{4}$ These findings mean that PEX syndrome is a general disorder of the extracellular matrix. ${ }^{4}$
A number of recent reports have suggested that PEX syndrome is related to many cardiovascular and cerebrovascular diseases such as transient ischaemic attacks, ${ }^{11}$ a history of angina pectoris, arterial hypertension, MI, or stroke, ${ }^{12}$ aneurysms of the abdominal aorta, ${ }^{13}$ and asymptomatic myocardial dysfunction. ${ }^{14}$

In their study, Citirik et $a l^{5}$ reported that the prevalence of PEX syndrome was significantly higher in patients with documented CAD, and the prevalence of CAD was also significantly higher in patients with PEX syndrome. In the other study, PEX syndrome has been found to be positively associated with the risk for CAD among patients with age $\geq 50$, and it has been suggested that PEX syndrome is a risk factor for CAD. ${ }^{6}$ However, Tarkkanenet $a l^{15}$ have also reported that the frequency of documented CAD was similar between the patients with primary open-angle and exfoliation glaucoma.

TDI at rest is useful in the evaluation of myocardial ischaemia and a tool for the quantification of ischaemiainduced regional myocardial dysfunction. It is expected that the $\mathrm{S}$ velocity, Ea velocity, and $\mathrm{Ea} / \mathrm{Aa}$ ratio decrease, and the ratio of $\mathrm{E} / \mathrm{Ea}$ increases in patients with myocardial ischaemia when compared with controls., ${ }^{9,16}$ A combination of TDI with stress has not become a part of echocardiography practice and has essential

shortcomings such as velocities, which vary by location, and image quality.

In previous studies, the investigators evaluated the relation between PEX syndrome and CAD in the patients who had ischaemic symptoms or documented CAD only. ${ }^{5,6}$ In our study, we investigated whether or not PEX syndrome is associated with subclinical myocardial ischaemia by means of the use of TDI in patients with no documented CAD.

We found that the S-wave velocity measured at all of the annuluses, the Ea velocity at the septal annulus, and the ratio of $\mathrm{Ea} / \mathrm{Aa}$ at the lateral annulus were significantly lower in patients with PEX syndrome. We suggest that these findings may indicate subclinical myocardial ischaemia in patients with PEX syndrome.

We found that although not statistically significant, BMI was lower in the study group. This may be rather counterintuitive, as cardiovascular morbidity is normally associated with a higher BMI.

In conclusion, our data reveal that there may be an association between PEX syndrome and subclinical myocardial ischaemia. Thus, we suggest that ophthalmologists should consider informing their PEX syndrome patients' general practitioners about a possible cardiac risk, and more detailed examination in terms of myocardial ischaemia may be done in patients with PEX syndrome who do not have overt CAD for early diagnosis. Additional studies with greater number of PEX patients are needed to clarify this relationship. 


\section{Study limitations}

This is a cross-sectional study, which is to determine the association between PEX syndrome and myocardial ischemia at one point of time. Thus, this study cannot provide definite information about causality. In this study, we could not present the evaluation of myocardial ischaemia with any other method, because many of the patients stayed at the submaximal stage during the exercise stress testing. In addition, our study had a relatively small study population.

\section{Summary}

\section{What was known before}

- PEX syndrome is related to many cardiovascular and cerebrovascular diseases.

- In previous studies, the investigators evaluated the relation between PEX syndrome and coronary artery disease in the patients who had ischaemic symptoms only, or who had been administered coronary angiography only.

What this study adds

- We assessed the relation between PEX syndrome and subclinical myocardial ischemia in the patients who had no documented CAD.

- Our data reveal that there may be an association between PEX syndrome and subclinical myocardial ischemia.

- Thus, we suggest that more detailed examination in terms of coronary ischemia may be done in patients who do not have overt CAD for early diagnosis.

\section{Conflict of interest}

The authors declare no conflict of interest.

\section{References}

1 Ritch R, Schlötzer-Schrehardt U. Exfoliation syndrome. Surv Ophthalmol 2001; 45(4): 265-315.

2 Vesti E, Kivela T. Exfoliation syndrome and exfoliation glaucoma. Prog Retin Eye Res 2000; 19(3): 345-368.

3 Streeten BW, Li ZY, Wallace RN, Eagle RC, Keshgegian AA. Pseudoexfoliative fibrillopathy in visceral organs of a patient with pseudoexfoliation syndrome. Arch Ophthalmol 1992; 110(12): 1757-1762.

4 Schlotzer-Schrehardt U, Naumann GO. Ocular and systemic pseudoexfoliation syndrome. Am J Ophthalmol 2006; 141(5): 921-937.
5 Citirik M, Acaroglu G, Batman C, Yildiran L, Zilelioglu O. A possible link between the pseudoexfoliation syndrome and coronary artery disease. Eye 2007; 21(1): 11-15.

6 Andrikopoulos GK, Mela EK, Georgakopoulos CD, Papadopoulos GE, Damelou AN, Alexopoulos DK et al. Pseudoexfoliation syndrome prevalence in Greek patients with cataract and its association to glaucoma and coronary artery disease. Eye 2009; 23(2): 442-447.

7 Thibault H, Derumeaux G. Assessment of myocardial ischemia and viability using tissue Doppler and deformation imaging: the lessons from the experimental studies. Arch Cardiovasc Dis 2008; 101(1): 61-68.

8 Derumeaux G, Ovize M, Loufoua J, Pontier G, André-Fouet $X$, Cribier A. Assessment of nonuniformity of transmural myocardial velocities by color-coded tissue Doppler imaging: characterization of normal, ischemic, and stunned myocardium. Circulation 2000; 101(12): 1390-1395.

9 Derumeaux G, Ovize M, Loufoua J, André-Fouet X, Minaire Y, Cribier A et al. Doppler tissue imaging quantitates regional wall motion during myocardial ischemia and reperfusion. Circulation 1998; 97(19): 1970-1977.

10 Schiller NB, Shah PM, Crawford M, DeMaria A, Devereux R, Feigenbaum $\mathrm{H}$ et al. Recommendations for quantitation of the left ventricle by two-dimensional echocardiography: American Society of Echocardiography committee on standards, subcommittee on quantitation of two-dimensional echocardiograms. J Am Soc Echocardiogr 1989; 2: 358-367.

11 Repo LP, Teräsvirta ME, Koivisto KJ. Generalized transluminance of the iris and the frequency of the pseudoexfoliation syndrome in the eyes of transient ischemic attack patients. Ophthalmology 1993; 100(3): 352-355.

12 Mitchell P, Wang JJ, Smith W. Association of pseudoexfoliation syndrome with increased vascular risk. Am J Ophthalmol 1997; 124(5): 685-687.

13 Schumacher S, Schlotzer-Schrehardt U, Martus P, Lang W, Naumann GO. Pseudoexfoliation syndrome and aneurysms of the abdominal aorta. Lancet 2001; 357(9253): 359-360.

14 Bojic L, Ermacora R, Polic S, Ivanisevic M, Mandic Z, Rogosic $\mathrm{V}$ et al. Pseudoexfoliation syndrome and asymptomatic myocardial dysfunction. Graefes Arch Clin Exp Ophthalmol 2005; 243(5): 446-449.

15 Tarkkanen A, Reunanen A, Kivela T. Frequency of systemic vascular diseases in patients with primary open-angle glaucoma and exfoliation glaucoma. Acta Ophthalmol 2008; 86(6): 598-602.

16 Lee KW, Blann AD, Lip GY. Impaired tissue Doppler diastolic function in patients with coronary artery disease: relationship to endothelial damage/ dysfunction and platelet activation. Am Heart J 2005; 150: 756-766. 